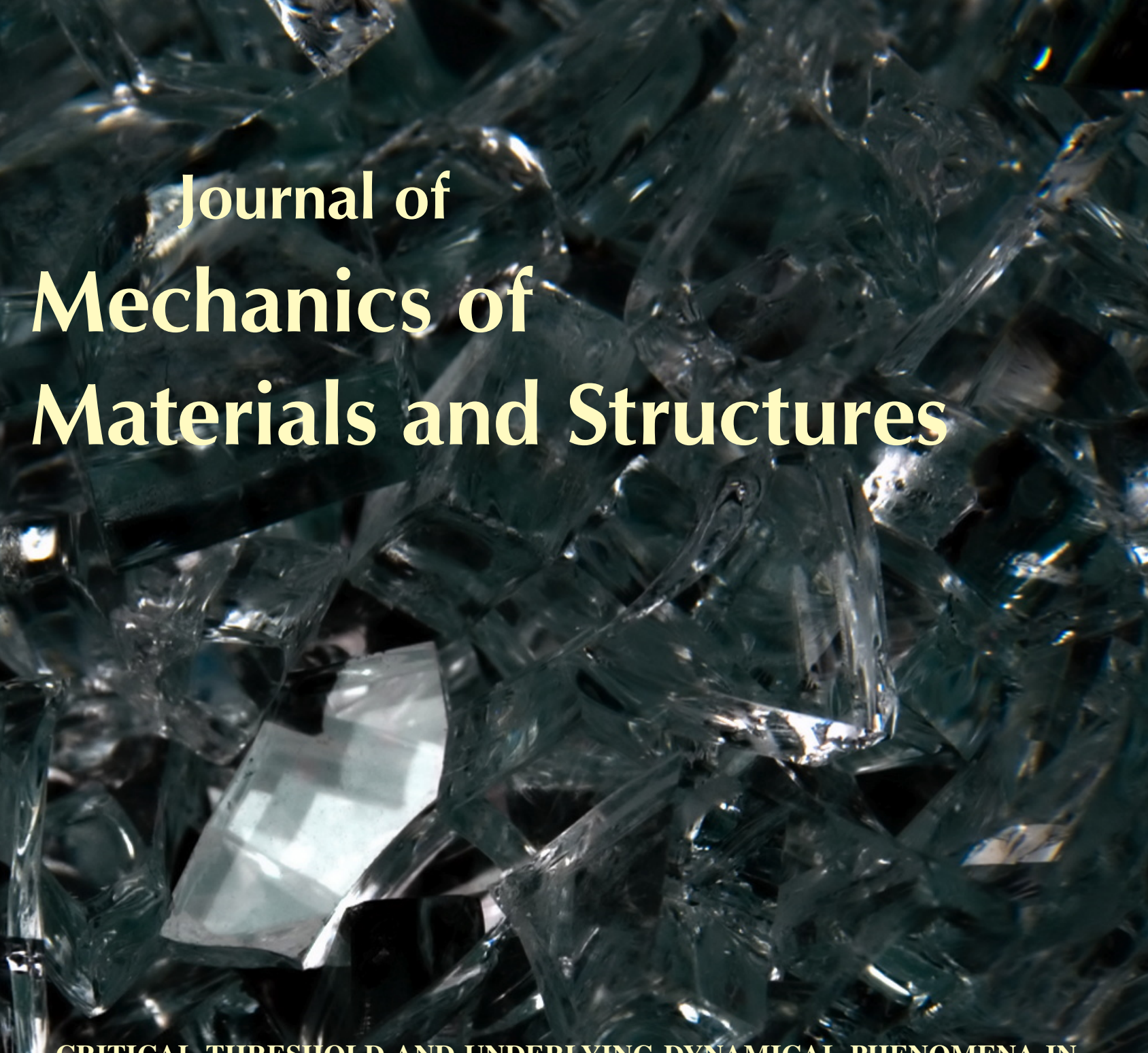




\title{
CRITICAL THRESHOLD AND UNDERLYING DYNAMICAL PHENOMENA IN PEDESTRIAN-INDUCED LATERAL VIBRATIONS OF FOOTBRIDGES
}

\author{
Stefano LENCI AND LAURA MARCHEGGIANI
}

\begin{abstract}
The problem of lateral vibrations of footbridges due to the synchronization of the pedestrians' motion with that of the supporting structure is analyzed by means of a 3D discrete time model. The map is linear in the mechanical part, and nonlinear in the synchronization part. A very simple and predictive formula is obtained for the critical number of pedestrians, which also takes into account the imperfect resonance between the pedestrians' natural frequencies and the bridge frequency. It is shown that the underlying mechanism triggering the sudden appearance of swaying bridge motion is a perturbation of a pitchfork bifurcation. The results presented in this paper are not related to a specific real case and are based on a quite reasonable hypothesis, and therefore it is expected that they have general validity.
\end{abstract}

\section{Introduction}

As a consequence of the technological development of new materials and of the architectural trend toward lightness and slenderness, modern footbridges have small natural frequencies, which can resonate with those of the pedestrian-induced load [Živanović et al. 2005; Venuti and Bruno 2009], which are in the range $1.4-2.4 \mathrm{~Hz}$ for vertical forcing and in the range $0.7-1.2 \mathrm{~Hz}$ for horizontal (lateral) forcing. In this situation unwanted large bridge motions may occur.

Various footbridges have experienced excessive lateral vibrations due to pedestrian-induced loads; the most famous is the London Millennium Bridge, which underwent, on its opening day, large horizontal vibrations due to the synchronization of the pedestrians' motion with the natural modes of the structure [Dallard et al. 2001a; 2001b]. Other bridges which have suffered similar problems are the Toda Park Bridge [Fujino et al. 1993; Nakamura and Kawasaki 2006] and the Maple Valley Bridge [Nakamura and Kawasaki 2006] in Japan, the Solferino Footbridge in Paris [Danbon and Grillaud 2005], and the Alexandra Bridge in Ottawa [Dallard et al. 2001a].

The pedestrian-induced lateral vibrations occurred in bridges of different structural types (suspension, cable-stayed, and steel girder bridges) as well as on footbridges made of different materials (steel, composite steel-concrete, and reinforced and prestressed concrete) [Živanović et al. 2005]. It is therefore confirmed that a large-enough crowd of pedestrians can induce strong lateral vibrations on footbridges of any type, although this requires the lateral mode to have a low-enough natural frequency [Dallard et al. 2001a], approximately below $1.2 \mathrm{~Hz}$, as stated.

The phenomenon behind pedestrian-induced lateral vibrations on footbridges is that of synchronous lateral excitation [Dallard et al. 2001a; Strogatz et al. 2005; Živanović et al. 2005; Eckhardt et al. 2007]. People walking in a crowd exhibit a random level of synchrony, and in general produce a lateral force on the bridge. In fact, even if the bridge is still and the pedestrians are not synchronized at all, due to the

Keywords: synchronization, discrete time model, Millennium Bridge, lateral induced vibrations, bridge-pedestrian coupling. 
stochasticity of the process the net force, which is the sum of all the lateral forces applied to the bridge by the footsteps of pedestrians, is not null, although it is possibly small. This small force produces small oscillations of the bridge.

As soon as the small bridge vibrations become perceptible to the unconscious human cognition processes, pedestrians tend spontaneously to walk in synchrony with the bridge, by slightly changing their walking frequency and phase. Of course, this tendency is somehow proportional to the vibration amplitude, and so it is very small, and possibly negligible, for very small displacements. However, it is believed that this phenomenon starts for very low levels of amplitude of the motion, well below the amplitude threshold perceived by conscious feeling.

This instinctive behavior, which is the mechanism through which the pedestrians interact with the bridge, produces an increase of the synchronization level, and the associated net force grows. This establishes an unwanted positive feedback loop, where the increase in oscillation amplitude causes pedestrians to increase their lateral footfall forcing and their level of synchrony, by following the movements of the deck in order to balance themselves [Dallard et al. 2001a; 2001b]: the more the bridge moves, the more the crowd pushes it to move further.

It has been observed that for potentially susceptible spans there is a critical number of pedestrians $N_{\text {cr }}$ that will cause the vibrations to increase suddenly to unacceptable levels. The oscillations are small below $N_{\text {cr }}$ and, due to the synchronization, they increase rapidly above $N_{\text {cr. }}$ This critical threshold is of great practical interest, and its prediction is the goal of almost all studies. This paper aims to provide a simple and reliable analytical prediction of $N_{\mathrm{cr}}$, as well as to further understanding of the overall phenomenon.

The nature of the problem is nonlinear, as has been confirmed, for example, by tests performed on the London Millennium Bridge [Dallard et al. 2001a]; in spite of this, however, it can be detected within a mechanically linear framework, since even the "large" oscillations are orders of magnitude smaller than the span length. The nonlinearity is only in the interaction between the structure and pedestrians.

Several papers have recently addressed this topic, even if a standard and generally accepted model of pedestrian-induced lateral dynamic loading and of dynamical interaction with the bridge is still missing. Živanović et al. [2005] have performed a comprehensive review of the existing literature on the topic until 2003, while an updated review can be found in [Venuti and Bruno 2009].

Early studies on pedestrian-induced vibrations of footbridges [Blanchard et al. 1977; Matsumoto et al. 1978; Wheeler 1980] concerned only the measurement and modeling of the vertical component of pedestrian load on a motionless surface.

Dallard et al. [2001a; 2001b] have conducted a series of controlled crowd tests on the Millennium Bridge and have proposed a load model based on empirical observations. Also a formula has been obtained for the critical number of pedestrians; it actually depends only on the modal damping of the bridge through a proportionality constant which is strictly related to the specific real case-study (the Millennium Bridge). The findings of the present paper extend somewhat these works, by better highlighting the nature of this constant (for example, that it depends on the bridge natural frequency).

Nakamura [2004] has proposed an interactive forcing model analogous to the previous one, but which allows the schematization of the self-limiting nature of the synchronization phenomenon and the prediction of the steady-state amplitude. Also this model is based on coefficients which have been estimated from experimental tests [Fujino et al. 1993; Nakamura and Kawasaki 2006] and cannot easily be generalized to other footbridges. 
In [Newland 2003] the problem is approached by referring to the interaction phenomenon between fluid flow and structures which is widely studied in wind engineering and commonly known as lock-in. His model includes the empirical assumption that $40 \%$ of the pedestrians are synchronized with the bridge lateral frequency, independent of the amplitude of the oscillations.

Fujino et al. [1993] have adopted a model of harmonic forcing by empirically tuning a synchronization parameter for the lateral vibrations of the Toda Park Bridge (according to their experimental data). This model does not predict any sudden transition to a vibrating state of the bridge but assumes a continuous increase in the vibration amplitude as the number of pedestrians increases.

Roberts [2005] has schematized the interaction between the pedestrians and the footbridge assuming that synchronization occurs when the pedestrians' motion is larger than the bridge motion; from this critical condition, he has obtained a limit number of pedestrians.

In [Ricciardelli and Pizzimenti 2007] a systematic experimental campaign has been performed aimed at characterizing dynamically the lateral force exerted by pedestrians on footbridges, both in the case of a still deck and in the case of a laterally moving deck; deterministic and stochastic lateral loading models for the static case have been provided and the bases have been put in place for more sophisticated dynamic models including crowd-structure interaction. The mechanism of crowd synchronization has been investigated only from the qualitative point of view, deferring quantitative study and modeling until after further measurements.

The excessive lateral vibrations of the Solferino Bridge in Paris have been explained in [Blekherman 2007] on the basis of autoparametric resonance by using a double pendulum model; the process of possible synchronization of pedestrian loading with the relevant vibrational modes, which are nonlinearly coupled in a ratio of 2:1 between their frequencies, depends on the achievement of parametric resonance.

Piccardo and Tubino [2008] have performed an interesting extensive critical analysis of the excitation mechanisms identified in the literature and they have proposed a new forcing model based on experimental tests carried out on harmonically moving platforms [Dallard et al. 2001a]. The force exerted by pedestrians is modeled as harmonic with an amplitude depending on the deck lateral displacement, and a simple criterion defining the limit pedestrian mass is introduced. They mainly ascribe to a mechanism of parametric excitation the lateral sway motion induced by crowds in very flexible, lowly damped footbridges, with a first lateral natural frequency around $0.5 \mathrm{~Hz}$ corresponding to half of the first lateral walking frequency.

In Venuti et al. [2007] a first-order model has been developed based on the mass conservation equation, in order to macroscopically describe the dynamics of the crowd in the framework of hydrodynamic modeling. The crowd, considered as a pedestrian flow, is assumed to behave like a continuous compressible fluid; the structural system is modeled by means of a generalized single degree of freedom (SDOF) model. The two-way interaction between the crowd and the structure is studied. This model permits taking into account the triggering of the lock-in and its self-limited nature, previously explained only in [Strogatz et al. 2005]. The effects of the two different kinds of synchronization, that is, between the pedestrians and the structure and among the pedestrians, are introduced; the presence of different frequency components in the overall force exerted by the pedestrians is considered. Some parameters, used in the formulation of the model, come from reasonable qualitative considerations about pedestrian behavior and would require specific experimental tests to be confirmed. 
In [Bodgi et al. 2007] a similar approach has been adopted to simulate the mechanics of synchronous lateral excitation induced by pedestrians on footbridges.

Strogatz et al. [2005] have been the first, to the best of our knowledge, to mathematically describe and predict the simultaneous growth of bridge movement and crowd synchronization, an observation that was unexplained in previous models but that is confirmed by analyses of video footage [Arup 2000] recorded during overcrowding conditions on real footbridges [Fujino et al. 1993; Dallard et al. 2001a]. They proposed a model (called SAMEO in [Marcheggiani and Lenci 2010] from the initials of the authors) which is particularly interesting for its contribution to the physical-mathematical explanation of the underlying mechanical event, as well as for the reasonable description of the phenomenon itself.

The SAMEO model is quite simple in its formulation and general enough to be possibly applied to any bridge at risk of synchronous lateral excitation. It models the bridge as a SDOF oscillator that interacts nonlinearly with each pedestrian. The pedestrians are modeled as limit-cycle phase oscillators (this choice comes from a similitude with biological systems, for example, fireflies). The key parameter of the model, $C$, measures the pedestrians sensitivity to bridge lateral vibrations; it can be determined only experimentally.

The SAMEO model has been investigated in depth in [Marcheggiani and Lenci 2010], where extensive numerical simulations have been performed in order to detect the effects of the main parameters on the system's response, in particular on the critical threshold. Various extensions have been proposed to model some important aspects not considered in [Strogatz et al. 2005], such as, for example, the self-interaction between pedestrians.

Although the original model and its extensions are simple in their formulation and meaning, they are quite involved in terms of the associated equations of motion, which is a set of $N+2(N$ being the number of pedestrians) nonlinear ordinary differential equations. This system can be fully solved only numerically, although some approximated analytical techniques have been obtained in [Abrams 2006] to get some partial information. This is a limitation of the model, together with the fact that it does not provide immediate information.

In order to overcome the previous drawbacks, in [Lenci and Marcheggiani 2008] a simplified model is proposed and applied with some success to the case of the Millennium Bridge. The main idea is that of passing from ordinary differential equations to maps, that is, from a continuous time system to a discrete time one. In particular, a peak-to-peak map [Candaten and Rinaldi 2000], similar to that introduced by Lorenz in discovering chaotic attractors, has been considered and analyzed in depth. The discrete time permits simple computations (which can be performed by hand), and provides a simple but very predictive formula for $N_{\mathrm{cr}}$ and a better understanding of the dynamical phenomena lurking in the background.

The work [Lenci and Marcheggiani 2008] is continued in this paper; another discrete time model is proposed, now based on the stroboscopic Poincaré map (instead of the peak-to-peak map). The mechanical part is described by the position $x$ and velocity $y$, while the bridge-pedestrian interaction is described by a new state variable $\sigma$ measuring the degree of synchronization of the pedestrians. We thus get a 3D map, linear in the mechanical part and nonlinear only in the interaction part, whose behavior is analyzed without exact knowledge of the evolution law for $\sigma$. Just its overall properties and local behavior are used, thus providing a very general analysis, which in particular extends that of [Lenci and Marcheggiani 
2008]. The main results are obtained by a bifurcation analysis of the fixed points of the map, which of course is specific to the considered simplified model.

This paper is organized as follows. In Section 2 the mechanical model is illustrated leading to the mechanical part of the discrete time model. Then, the pedestrian-bridge interaction is analyzed in Section 3, where the main properties of the third evolution law are discussed. The fixed points of the map, which are the dynamical behaviors of interest for the computation of the critical threshold, are considered in Section 4, where a simple formula for $N_{\mathrm{cr}}$ is obtained, and where the effect of imperfections is discussed. Some properties of the map in the resonant case are discussed in Section 5, and conclusions are presented in Section 6.

\section{Mechanical model}

Based on experience in real cases, in particular on that of the London Millennium Bridge mentioned in Section 1, we assume that the phenomenon of lateral synchronization involves only one lateral mode $\varphi(Z)$ of the structure, so that the mechanical equation of motion is

$$
M \ddot{X}(T)+B \dot{X}(T)+K X(T)=F(T),
$$

where $M, B$, and $K$ are the modal mass, damping, and stiffness, respectively, and $X(T)$ is the modal amplitude. Note that $M$ includes also the mass of the pedestrians, and in general is not a fixed number. However, in real cases the mass of pedestrians is about $10-15 \%$ of the total mass, and so it is expected that it does not play a key role. $F(T)$ is the modal force, that is, the projection on the considered mode $\varphi(Z)$ of the force $\bar{F}(Z, T)$ exerted by pedestrians along the span, $F(T)=\int_{0}^{L} \bar{F}(Z, T) \varphi(Z) d Z$. In fact, the load of each pedestrian depends not only on the force he applies on the bridge, but also on his position $Z \in[0, L]$ along the span.

The definitions

$$
\Omega=\sqrt{\frac{K}{M}}, \quad t=\Omega T, \quad \xi=\frac{B}{2 \sqrt{M K}}=\frac{B \Omega}{2 K}, \quad x(t)=X(T), \quad f(t)=\frac{F(T)}{K},
$$

where $\Omega$ is the natural frequency of the considered mode, permit us to rewrite (1) in the form

$$
\ddot{x}(t)+2 \xi \dot{x}(t)+x(t)=f(t),
$$

which will be used in the following. Note that the time $t$ is dimensionless, while $x$ has the dimension of length.

2.1. A single pedestrian and the stroboscopic Poincaré map. We initially consider the effect of a single pedestrian by assuming

$$
f(t)=g \sin \left(\omega_{p} t-\phi\right)
$$

where:

- $g>0$ is the dimensionless amplitude, such that $G=g K \cong 30 N$ is the maximum lateral force exerted by a pedestrian [Belli et al. 2001; Marcheggiani and Lenci 2010];

- $\omega_{p}$ is the dimensionless (circular) frequency, such that $f_{p}=\Omega_{p} /(2 \pi)=\Omega \omega_{p} /(2 \pi)=0.7-1.2 \mathrm{~Hz}$ is the pedestrian footstep native frequency [Živanović et al. 2005]; and 
- $\phi \in[0,2 \pi]$ is the pedestrian phase, which depends on the time the pedestrian enters the bridge.

Equation (4) is an approximation of the real force, since experimental data concerning lateral walking forces on a still surface [Bodgi et al. 2007; Ricciardelli and Pizzimenti 2007] have shown that it is much closer to a square wave than to a harmonic force [Belli et al. 2001]. However, expression (4) can be considered as the first term in the Fourier series of the real excitation, thus capturing the most important energy content and maintaining the simple expression needed for analytical computations.

The solution of (3) and (4) starting from $x(0)=x_{n}$ and $\dot{x}(0)=y_{n}$ is

$$
x(t)=e^{-\xi t}\left[c_{1} \sin \left(t \sqrt{1-\xi^{2}}\right)+c_{2} \cos \left(t \sqrt{1-\xi^{2}}\right)\right]+g\left[d_{1} \sin \left(\omega_{p} t\right)+d_{2} \cos \left(\omega_{p} t\right)\right],
$$

where

$$
\begin{aligned}
d_{1} & =\frac{\left(1-\omega_{p}^{2}\right) \cos (\phi)-2 \xi \omega_{p} \sin (\phi)}{\left(1-\omega_{p}^{2}\right)^{2}+\left(2 \xi \omega_{p}\right)^{2}}, & d_{2} & =\frac{-\left(1-\omega_{p}^{2}\right) \sin (\phi)-2 \xi \omega_{p} \cos (\phi)}{\left(1-\omega_{p}^{2}\right)^{2}+\left(2 \xi \omega_{p}\right)^{2}}, \\
c_{1} & =\frac{\xi x_{n}+y_{n}-\omega_{p} g d_{1}-\xi g d_{2}}{\sqrt{1-\xi^{2}}}, & c_{2} & =-g d_{2}+x_{n} .
\end{aligned}
$$

Note that the initial conditions do not modify $d_{1}$ and $d_{2}$, only $c_{1}$ and $c_{2}$.

After one period $T_{p}=2 \pi / \omega_{p}$ of the excitation we have from (5)

$$
x\left(T_{p}\right)=e^{-\xi T_{p}}\left[c_{1} \sin \left(T_{p} \sqrt{1-\xi^{2}}\right)+c_{2} \cos \left(T_{p} \sqrt{1-\xi^{2}}\right)\right]+g d_{2}
$$

and

$$
\dot{x}\left(T_{p}\right)=e^{-\xi T_{p}}\left[\left(-c_{1} \xi-c_{2} \sqrt{1-\xi^{2}}\right) \sin \left(T_{p} \sqrt{1-\xi^{2}}\right)+\left(-c_{2} \xi+c_{1} \sqrt{1-\xi^{2}}\right) \cos \left(T_{p} \sqrt{1-\xi^{2}}\right)\right]+g \omega_{p} d_{1} .
$$

The main idea of this paper consists in moving from a continuous time system, (3), to a discrete one. This can be obtained by introducing an appropriate Poincare section of the continuous flow, and by considering the associated Poincaré return map [Wiggins 1997].

We use the stroboscopic Poincare map obtained by sampling the system position and velocity at each excitation period $T_{p}=2 \pi / \omega_{p}$, which is mathematically well defined. It is given by

$$
\left\{\begin{array}{l}
x_{n+1} \\
y_{n+1}
\end{array}\right\}=\left\{\begin{array}{l}
f_{x}\left(x_{n}, y_{n}\right) \\
f_{y}\left(x_{n}, y_{n}\right)
\end{array}\right\}=e^{-\xi T_{p}}\left[\begin{array}{cc}
\alpha_{x} & \alpha_{x y} \\
-\alpha_{x y} & \alpha_{y}
\end{array}\right]\left\{\begin{array}{l}
x_{n} \\
y_{n}
\end{array}\right\}+g\left\{\begin{array}{l}
\beta_{x} \\
\beta_{y}
\end{array}\right\}
$$

where use is made of (7) and (8), and where $x_{n+1}=x\left(T_{p}\right)$ and $y_{n+1}=\dot{x}\left(T_{p}\right)$ (see [Wiggins 1997]), the functions $f_{x}\left(x_{n}, y_{n}\right)$ and $f_{y}\left(x_{n}, y_{n}\right)$ are defined by the last equality, and

$$
\begin{aligned}
\alpha_{x} & =\xi \frac{\sin \left(T_{p} \sqrt{1-\xi^{2}}\right)}{\sqrt{1-\xi^{2}}}+\cos \left(T_{p} \sqrt{1-\xi^{2}}\right), & & \alpha_{y}=-\xi \frac{\sin \left(T_{p} \sqrt{1-\xi^{2}}\right)}{\sqrt{1-\xi^{2}}}+\cos \left(T_{p} \sqrt{1-\xi^{2}}\right), \\
\alpha_{x y} & =\frac{\sin \left(T_{p} \sqrt{1-\xi^{2}}\right)}{\sqrt{1-\xi^{2}}}, & & \\
\beta_{x} & =e^{-\xi T_{p}}\left(-\omega_{p} d_{1} \alpha_{x y}-d_{2} \alpha_{x}\right)+d_{2}, & \beta_{y} & =e^{-\xi T_{p}}\left(-\omega_{p} d_{1} \alpha_{y}+d_{2} \alpha_{x y}\right)+\omega_{p} d_{1} .
\end{aligned}
$$

Note that in the resonant case $T_{p}=2 \pi / \sqrt{1-\xi^{2}}$ we have $\alpha_{x}=\alpha_{y}=1, \alpha_{x y}=0, \beta_{x}=d_{2}\left(1-e^{-\xi T_{p}}\right)$, and $\beta_{y}=\omega_{p} d_{1}\left(1-e^{-\xi T_{p}}\right)$. 
2.2. Crowd of pedestrians. When a crowd of $N$ uniformly distributed pedestrians is walking on the bridge the net force is

$$
f(t)=\sum_{i=1}^{N} g_{i} \sin \left(\omega_{p, i} t-\phi_{i}\right)
$$

In principle, the parameters $g_{i}$ and $\omega_{p, i}$ are stochastic variables which depend on the age, health condition, height, etc., of the population of pedestrians. However, $\phi_{i}$, which is also a stochastic variable, depends only on the instant of time the pedestrian enters the bridge, and not on his human characteristics.

Single pedestrian action is modeled by (4) and the action of the crowd by (11); we neglect the interactions between pedestrians, and focus only on the interaction of each pedestrian with the bridge, which is the main mechanism responsible for the considered phenomenon. For the pedestrian-pedestrian interactions, an interesting topic involving complex living systems, but which is out of the scope of this paper, we refer to, for example, [Johansson et al. 2008].

In the following we make the assumption that each pedestrian of the crowd has the same natural frequency, $\omega_{p, i}=\omega_{p}$. This is motivated by the fact that only pedestrians with a natural frequency close to that of the bridge can undergo the synchronization phenomenon we are dealing with, since it involves resonance. This fact is confirmed by the movie of the opening of the Millennium Bridge [Arup 2000], where it is clearly seen that only some pedestrians synchronize (it was estimated at about $40 \%$ [Newland 2003]). The others are not influenced by the bridge motion and maintain their natural walking, and so, by stochastic arguments, we can assume that they provide a zero net force on the bridge and thus are not of interest. We conclude that only a narrow band of native frequencies is of real interest, and we consider just one, $\omega_{p}$, in order to fulfill the objective of having a simple, but predictive, model.

By the previous basic hypothesis, which guarantees that the stroboscopic Poincaré map is still well defined, we have that (11) becomes

$$
f(t)=\sin \left(\omega_{p} t\right) \sum_{i=1}^{N} g_{i} \cos \left(\phi_{i}\right)-\cos \left(\omega_{p} t\right) \sum_{i=1}^{N} g_{i} \sin \left(\phi_{i}\right) .
$$

The summations appearing in (12) depend on the degree of synchronization of the pedestrians, that is, on the degree of correlation of their phases $\phi_{i}$.

In the case of perfectly asynchronous pedestrians we have that $\phi_{i}$ is a stochastic variable uniformly distributed in $[0,2 \pi]$, which implies that

$$
\sum_{i=1}^{N} g_{i} \cos \left(\phi_{i}\right)=\sum_{i=1}^{N} g_{i} \sin \left(\phi_{i}\right)=0 \Rightarrow f(t)=0 .
$$

This can be seen by a standard Monte Carlo analysis. In practice in this case for each pedestrian there exists, on average, a pedestrian with opposite phase.

In the perfectly synchronous case pedestrians have exactly the same phase, $\phi_{i}=\phi \pm 2 n \pi$, so that

$$
\sum_{i=1}^{N} g_{i} \cos \left(\phi_{i}\right)=\cos (\phi) \sum_{i=1}^{N} g_{i}=\cos (\phi) N g_{\mathrm{av}}, \quad \sum_{i=1}^{N} g_{i} \sin \left(\phi_{i}\right)=\sin (\phi) \sum_{i=1}^{N} g_{i}=\sin (\phi) N g_{\mathrm{av}},
$$


and

$$
f(t)=N g_{\text {av }} \sin \left(\omega_{p} t-\phi\right) .
$$

In the previous expressions $N$ is the number of (synchronized) pedestrians whose frequency is close to $\omega_{p}$, that is, a subset of the total number of pedestrians walking on the bridge (see previous comments); only in calibrated experiments with controlled people is $N$ the total number of pedestrians. The average force of each pedestrian is $g_{\mathrm{av}}$, such that $G_{\mathrm{av}}=g_{\mathrm{av}} K \cong 30 N$ (see Section 2.1), and $\phi$ is the average phase; its value is inessential, and it will be used in due course to simplify the computations.

From the previous expressions we see that $f(t)$ ranges from $f(t)=0$ (the perfectly asynchronous case) to $f(t)=N g_{\text {av }} \sin \left(\omega_{p} t-\phi\right)$ (the perfectly synchronous case). In real cases the actual force is in between these two bounds, and depends on the degree of synchronization. Thus we assume

$$
f(t)=N g_{\text {av }} \sigma \sin \left(\omega_{p} t-\phi\right),
$$

where $\sigma$ is a dimensionless measure of the degree of synchronization, which ranges from 0 (the perfectly asynchronous case) to 1 (the perfectly synchronous case).

Equation (16) is formally identical to (4), so that mathematically we bring back the crowd case to that of an equivalent (single) pedestrian, and we can take advantage of the formulas of Section 2.1. In doing this, we use the "free" overall phase to simplify the expressions. In particular, by assuming (without loss of generality)

$$
\sin (\phi)=\frac{-2 \xi \omega_{p}}{\sqrt{\left(1-\omega_{p}^{2}\right)^{2}+\left(2 \xi \omega_{p}\right)^{2}}}, \quad \cos (\phi)=\frac{1-\omega_{p}^{2}}{\sqrt{\left(1-\omega_{p}^{2}\right)^{2}+\left(2 \xi \omega_{p}\right)^{2}}}
$$

we have

$$
d_{1}=\frac{1}{\sqrt{\left(1-\omega_{p}^{2}\right)^{2}+\left(2 \xi \omega_{p}\right)^{2}}}, \quad d_{2}=0,
$$

so that

$$
\beta_{x}=-\omega_{p} d_{1} e^{-\xi T_{p}} \alpha_{x y}, \quad \beta_{y}=\omega_{p} d_{1}\left(-e^{-\xi T_{p}} \alpha_{y}+1\right) .
$$

The map (9) becomes

$$
\left\{\begin{array}{l}
x_{n+1} \\
y_{n+1}
\end{array}\right\}=e^{-\xi T_{p}}\left[\begin{array}{cc}
\alpha_{x} & \alpha_{x y} \\
-\alpha_{x y} & \alpha_{y}
\end{array}\right]\left\{\begin{array}{l}
x_{n} \\
y_{n}
\end{array}\right\}+\sigma \bar{N}\left\{\begin{array}{c}
-e^{-\xi T_{p}} \alpha_{x y} \\
1-e^{-\xi T_{p}} \alpha_{y}
\end{array}\right\},
$$

where

$$
\bar{N}=\frac{N g_{\mathrm{av}} \omega_{p}}{\sqrt{\left(1-\omega_{p}^{2}\right)^{2}+\left(2 \xi \omega_{p}\right)^{2}}} .
$$

\section{Pedestrian-bridge interaction}

In the previous section only the mechanical part has been considered. In order to model the dynamical bridge-pedestrian interaction and to describe the natural tendency of the systems to synchronize, we must consider also the human part, starting from the basic observation that the two parts influence each other. 
The first step in this direction is to assume that not only $x_{n}$ and $y_{n}$ vary in (discrete) time, but also the synchronization parameter $\sigma$, which is now considered as a state variable, $\sigma_{n}$, and no longer as a (fixed) parameter. Thus, (20) becomes

$$
\left\{\begin{array}{l}
x_{n+1} \\
y_{n+1}
\end{array}\right\}=\left\{\begin{array}{l}
f_{x}\left(x_{n}, y_{n}, \sigma_{n}\right) \\
f_{y}\left(x_{n}, y_{n}, \sigma_{n}\right)
\end{array}\right\}=e^{-\xi T_{p}}\left[\begin{array}{cc}
\alpha_{x} & \alpha_{x y} \\
-\alpha_{x y} & \alpha_{y}
\end{array}\right]\left\{\begin{array}{l}
x_{n} \\
y_{n}
\end{array}\right\}+\sigma_{n} \bar{N}\left\{\begin{array}{c}
-e^{-\xi T_{p}} \alpha_{x y} \\
1-e^{-\xi T_{p}} \alpha_{y}
\end{array}\right\} .
$$

Note that the passage from (20) to (22) is not a simple substitution of $\sigma$ with $\sigma_{n}$, but a conceptual change which, for example, increases the dimension of the dynamical system.

The next step consists in proposing a (discrete time) evolution law for the new state variable $\sigma_{n}$ :

$$
\sigma_{n+1}=f_{\sigma}\left(x_{n}, y_{n}, \sigma_{n}\right),
$$

so that (22) and (23) become a well-defined dynamical system. The choice of the function $f_{\sigma}\left(x_{n}, y_{n}, \sigma_{n}\right)$ entails modeling the bridge-pedestrian interaction, and so it is the key point. In fact, while for the mechanical part (22) there are physical (Newtonian) laws, for the human part (23) there are no corresponding axiomatic laws, and any choice is by definition subjective.

Common sense suggests that the degree of synchronization strongly depends on the amplitude,

$$
A_{n}=\sqrt{x_{n}^{2}+\frac{y_{n}^{2}}{\omega_{p}^{2}}}
$$

of the bridge motion, and weakly on the current synchronization $\sigma_{n}$. Thus, in this work we assume

$$
\sigma_{n+1}=f_{\sigma}\left(A_{n}\right)
$$

The following properties help in the characterization of the nonlinear function $f_{\sigma}\left(A_{n}\right)$ :

(1) $f_{\sigma}(0)=0$. In fact, in a (mathematically) perfect case, in the absence of motion there is no synchronization at all and the force on the bridge is zero. Actually, since the synchronization is a stochastic process, in real (or imperfect) cases even if the bridge is still, the lack of synchronization is not perfect, and there is a net force, although very small. This is achieved by assuming $f_{\sigma}(0)=\varepsilon$, $|\varepsilon| \ll 1$. In the sequel we will consider both the perfect and the imperfect cases.

(2) $f_{\sigma}\left(A_{n}\right)$ is a monotonic increasing function, since there is experimental evidence that the degree of synchronization increases with the amplitude of the motion.

(3) $f_{\sigma}\left(A_{n}\right)$ is as simple as possible, since there is no experimental evidence for strange behaviors for certain values of $A_{0}$. Mathematically this property can be formulated by assuming that $f_{\sigma}\left(A_{n}\right)$ is smooth, that is, continuously differentiable, in $] 0, \infty[$ and that it has at maximum one inflection point.

(4) $\lim _{A_{n} \rightarrow \infty} f_{\sigma}\left(A_{n}\right)=1$, as, for large excitation amplitudes, all the pedestrians synchronize (that is, there is no asymptotic limit less than 1). This property mathematically describes the saturation condition; in practice the rate of convergence toward 1 is important, since it is practically expected that for large but finite values of $A_{n}$ we have achieved a practically complete synchronization.

Any function satisfying the previous four points is acceptable in principle. 
For the forthcoming developments the most important characteristic of the function $f_{\sigma}\left(A_{n}\right)$ is its behavior around the origin $A_{n}=0$. Without loss of generality we can assume the following local behavior:

$$
f_{\sigma}\left(A_{n}\right)=\varepsilon+\gamma_{k}\left(A_{n}\right)^{k}+\ldots,
$$

where $\varepsilon$ is the imperfections parameter (see point (1)), $k$ is a positive real number determining the local rate of convergence toward $A_{n}=0$, and $\gamma_{k}$ is a parameter measuring the "slope" of the local behavior, that is, the sensitivity of the pedestrians to the movement of the bridge. Both $k$ and $\gamma_{k}$ are parameters of the model to be determined theoretically or experimentally.

\section{Fixed points}

Now that we have the map ((22) and (25)) describing the evolution law for the coupled bridge-pedestrian system we can study its dynamic behavior. We start by considering the fixed points

$$
x_{0}=f_{x}\left(x_{0}, y_{0}, \sigma_{0}\right), \quad y_{0}=f_{y}\left(x_{0}, y_{0}, \sigma_{0}\right), \quad \sigma_{0}=f_{\sigma}\left(A_{0}\right), \quad A_{0}=\sqrt{x_{0}^{2}+\frac{y_{0}^{2}}{\omega_{p}^{2}}},
$$

which correspond to periodic oscillations of the original continuous time system.

Solving the first two equations of (27) yields

$$
x_{0}=0, \quad y_{0}=\sigma_{0} \bar{N} \quad \Rightarrow \quad A_{0}=\frac{\sigma_{0} \bar{N}}{\omega_{p}} .
$$

Substituting this expression in $(27)_{3}$ gives the nonlinear algebraic equations permitting determination of the fixed points:

$$
\sigma_{0}=f_{\sigma}\left(\frac{\sigma_{0} \bar{N}}{\omega_{p}}\right) \Rightarrow \frac{\omega_{p}}{\bar{N}} A_{0}=f_{\sigma}\left(A_{0}\right) .
$$

Equation (29) can be graphically solved by drawing the graph of $f_{\sigma}\left(A_{0}\right)$ and of the straight line $\left(\omega_{p} / \bar{N}\right) A_{0}$, as schematically shown in Figure 1 . This permits the inference of the main qualitative properties of the solution without exact knowledge of the function $f_{\sigma}\left(A_{0}\right)$.

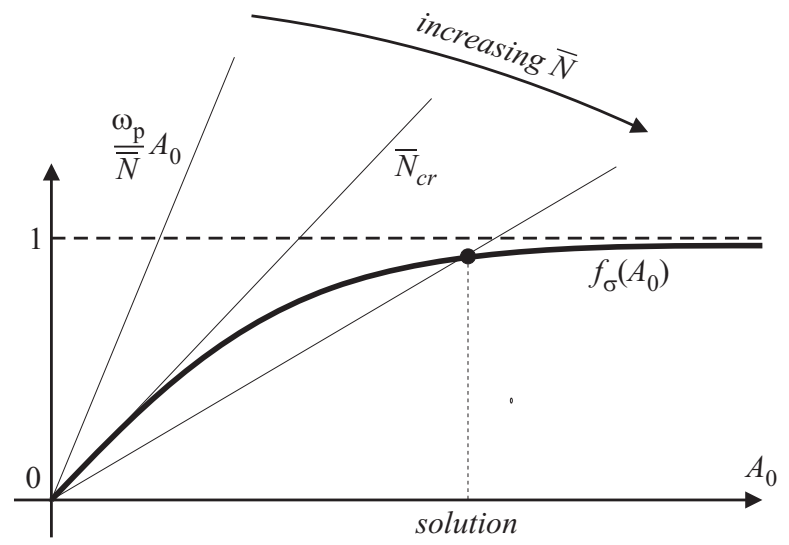

Figure 1. A schematic representation of the graphical solution of (29). 
The solutions of (29) are now discussed by considering separately the perfect $(\varepsilon=0$, Figure 1$)$ and imperfect $(\varepsilon \neq 0)$ cases, and by using $\bar{N}$ as a varying (driving) parameter for parametric analysis and for bifurcation diagrams.

4.1. Perfect case. In the perfect case $f_{\sigma}(0)=0$, so that we have the trivial (or rest) solution $A_{0}=0$ corresponding to the still bridge (see (29) and Figure 1). This is the main path of solutions.

To determine if there are secondary solutions bifurcating from the trivial one, we consider the local behavior (26) of $f_{\sigma}\left(A_{0}\right)$, so that (29) becomes

$$
\frac{\omega_{p}}{\bar{N}} A_{0}=\gamma_{k} A_{0}^{k}+\ldots
$$

From the previous equation we conclude that if $k \neq 1$ there are no solutions in the neighborhood of $A_{0}=0$ for finite values of $\bar{N}$, that is, there are no bifurcation points.

If, on the other hand, $k=1$, then there is a branching at (we write $\gamma$ instead of $\gamma_{1}$ for simplicity)

$$
\bar{N}_{\mathrm{cr}}=\frac{\omega_{p}}{\gamma}
$$

which, as shown in Figure 1, corresponds to the $\bar{N}$ providing the same slope at the origin for $f_{\sigma}\left(A_{0}\right)$ and $\left(\omega_{p} / \bar{N}\right) A_{0}$.

Combining (21) and (31) we get

$$
N_{\mathrm{cr}}=\frac{\sqrt{\left(1-\omega_{p}^{2}\right)^{2}+\left(2 \xi \omega_{p}\right)^{2}}}{\gamma g_{\mathrm{av}}} .
$$

This expression is the most important result from a practical point of view, since it gives the critical number of pedestrians triggering the phenomenon of lateral synchronization, that is, the maximum number of (synchronizable) pedestrians allowed on the bridge deck. In fact, below this threshold there is only the rest solution, so nothing happens. It is just at this $N_{\mathrm{cr}}$ that a different solution becomes possible, and the swaying of the bridge appears. This is enough from a designer point of view, and it is valuable because (32) is a very simple formula obtained with reasonable hypotheses. In particular, it does not require knowledge of the whole function $f_{\sigma}\left(A_{0}\right)$, but only of its local behavior.

The model parameter $\gamma$, which has dimensions of inverse length, measures the sensitivity of the pedestrians to the bridge motion. Its meaning can be understood by considering the following piecewise linear expression, which is the simplest choice for $f_{\sigma}\left(A_{0}\right)$ :

$$
f_{\sigma}\left(A_{0}\right)= \begin{cases}\gamma A_{0}, & \text { for } 0<A_{0} \leq \frac{1}{\gamma}, \\ 1, & \text { for } A_{0} \geq \frac{1}{\gamma} .\end{cases}
$$

This expression shows that $1 / \gamma$ can be approximately considered as the amplitude such that all the synchronizable pedestrians are actually synchronized. In fact, the limit for $A_{n} \rightarrow \infty$ in point (4) is just a mathematical issue, since in practice the phenomenon occurs for small (or moderately small) displacements, justifying the mechanically linear framework used in (1).

Expression (32) provides the critical number as a function of the pedestrians' native frequency $\omega_{p}$. The worst situation corresponds to the resonant case, because in this case each pedestrian has the maximum 
effect on the bridge. In fact, by minimizing (32) with respect to $\omega_{p}$ we get

$$
\omega_{p, \min }=\sqrt{1-2 \xi^{2}}=\omega_{\mathrm{res}},
$$

confirming, as expected, that the resonance is the worst situation. By inserting (34) into (32) we obtain

$$
N_{\mathrm{cr}, \min }=\frac{2 \xi}{\gamma g_{\mathrm{av}}}=\frac{B}{\sqrt{M K}} \frac{1}{\gamma} \frac{K}{G_{\mathrm{av}}}=\frac{B \Omega}{\gamma G_{\mathrm{av}}},
$$

where we have assumed $4 \xi^{2}-4 \xi^{4} \cong 4 \xi^{2}$ due to the smallness of $\xi$, and where we remember that $G_{\text {av }} \cong 30 N$ [Belli et al. 2001; Marcheggiani and Lenci 2010].

Expression (35) is the same one obtained in [Lenci and Marcheggiani 2008] with a different model, and agrees with the predictions of the more sophisticated SAMEO model studied in [Marcheggiani and Lenci 2010]. The fact that it is a result of two different models supports its reliability. In fact, in [Lenci and Marcheggiani 2008] it has been shown that it predicts very well experimental results from the literature. Furthermore, based on the results of the London Millennium Bridge, it has been shown that a reasonable value for $\gamma$, likely valid in any circumstance, is $\gamma=0.14-0.17 \mathrm{~cm}^{-1}=14-17 \mathrm{~m}^{-1}$. This means that there is a complete synchronization for $\delta=1 / \gamma=6-7 \mathrm{~cm}$ ( $\delta$ refers to the [Lenci and Marcheggiani 2008] notation), a fact that agrees well with experimental observations [Arup 2000; Dallard et al. 2001a; 2001b]. This value also agrees well with the $4.5 \mathrm{~cm}$ identified as the limit lateral displacement in [Nakamura and Kawasaki 2006].

Expression (32) is the generalization of (35) to the case of nonperfect resonance, since, contrarily to (35), it permits the detection, still in a simple way, of the effects of $\omega_{p}$.

Formula (35) is now extremely simple, since it requires only the knowledge of the real damping and circular frequency of the involved (lateral) mode

$$
N_{\text {cr,min }}=0.0022 B\left[\mathrm{~kg} \mathrm{sec}^{-1}\right] \Omega\left[\mathrm{sec}^{-1}\right] .
$$

In spite of its straightforwardness, it is very predictive. In fact, we remember that the critical number of pedestrians which destabilized the north span of the London Millennium Bridge was about 155 [Dallard et al. 2001a; 2001b]. Since for the north span we have [Strogatz et al. 2005] $M=113000 \mathrm{~kg}, K=$ $4730000 \mathrm{~kg} \mathrm{sec}^{-2} \rightarrow \Omega=6.47 \mathrm{sec}^{-1}$ (that is, the natural frequency is $1.03 \mathrm{~Hz}$ ), and $B=11000 \mathrm{~kg} \mathrm{sec}^{-1}$, we obtain from (36) $N_{\mathrm{cr}, \min }=156$. Note that the mass of the critical number of pedestrians is about $m=155 \times 80=12480 \mathrm{~kg}$, that is, $11 \%$ of the modal mass.

To further show its reliability, we apply (36) to the Toda Park Bridge, best known as the T-Bridge, a cable-stayed footbridge in Japan. According to [Nakamura and Kawasaki 2006] we have $M=237000 \mathrm{~kg}$, $K=8092000 \mathrm{~kg} \mathrm{sec}^{-2} \rightarrow \Omega=5.84 \mathrm{sec}^{-1}$ (that is, the natural frequency is $0.93 \mathrm{~Hz}$ ), and $B=22200 \mathrm{~kg} \mathrm{sec}^{-1}$. Therefore the critical number of synchronizable pedestrians is $N_{\mathrm{cr}, \min }=285$. In this case we do not have the experimental value of $N_{\mathrm{cr}}$, as in the case of the Millennium Bridge, but we know from [Fujino et al. 1993 ] that with $N \cong 2000$ pedestrians (an extremely congested situation) the bridge experienced synchronized oscillations. Considering that about $20 \%$ of pedestrians synchronized, as explicitly remarked in [Fujino et al. 1993], we have $N \cong 400$, which is in good agreement with $N_{\mathrm{cr}, \min }=285$ (we cannot expect equality, since we have data only for a synchronized situation).

In the case of the Maple Valley cable-stay bridge, also known as the M-Bridge, in Japan, we have that the third asymmetric and, to a minor extent, the fourth symmetric modes are involved in the lateral 
synchronization [Nakamura and Kawasaki 2006; Nakamura and Kawasaki 2009]. For the third mode we have synchronization, for example, when there are about 41 pedestrians on the deck (see [Nakamura and Kawasaki 2009, case M-6, Figure 11]). In this case we have [Nakamura and Kawasaki 2009] $M=$ $97200 \mathrm{~kg}, K=29648570 \mathrm{~kg} \mathrm{sec}^{-2} \rightarrow \Omega=5.52 \mathrm{sec}^{-1}$ (that is, the natural frequency is $0.88 \mathrm{~Hz}$ ), and $B=2905 \mathrm{~kg} \mathrm{sec}^{-1}$. Therefore the critical number of synchronizable pedestrians is $N_{\mathrm{cr}, \min }=35$.

From the previous work we have seen that the main solution curve has a bifurcation point at $N_{\mathrm{cr}}$. The type of bifurcation depends on the higher-order terms of the Taylor expansion (26):

$$
f_{\sigma}\left(A_{0}\right)=\gamma A_{0}+\gamma_{2}\left(A_{0}\right)^{2}+\gamma_{3}\left(A_{0}\right)^{3}+\ldots,
$$

so that from (29) the local behavior of the branching solution is

$$
\bar{N}\left(A_{0}\right)=\frac{\omega_{p}}{\gamma}-\frac{\omega_{p} \gamma_{2}}{\gamma^{2}} A_{0}-\frac{\omega_{p}\left(\gamma_{3} \gamma-\gamma_{2}^{2}\right)}{\gamma^{3}}\left(A_{0}\right)^{2}+\ldots
$$

From (38) we see that if $\gamma_{2} \neq 0$ we have a transcritical bifurcation. Otherwise, we have a supercritical pitchfork bifurcation if $\gamma_{3}<0$ or a subcritical pitchfork bifurcation if $\gamma_{3}>0$ (in this case $f_{\sigma}\left(A_{0}\right)$ has an inflection point, which implies that the pitchfork is preceded by a saddle-node bifurcation for a lower value of $\bar{N}$, see Figure 2); this is a consequence of the fact that the trivial solution is stable for $N<N_{\mathrm{cr}}$. This is obvious by common sense, and can be proved mathematically by noticing that the Jacobian matrix of the map at the rest position is

$$
\left[\begin{array}{ccc}
\alpha_{x} e^{-\xi T_{p}} & \alpha_{x y} e^{-\xi T_{p}} & -\bar{N} \alpha_{x y} e^{-\xi T_{p}} \\
-\alpha_{x y} e^{-\xi T_{p}} & \alpha_{y} e^{-\xi T_{p}} & \bar{N}\left(1-\alpha_{y} e^{-\xi T_{p}}\right) \\
0 & \frac{\gamma}{\omega_{p}} & 0
\end{array}\right],
$$

and the associated characteristic equation is (use is made of the property $\alpha_{x} \alpha_{y}+\alpha_{x y}^{2}=1$ )

$$
s^{3}-e^{-\xi T_{p}}\left(\alpha_{x}+\alpha_{y}\right) s^{2}+\left(\frac{\gamma \bar{N}}{\omega_{p}}\left(-1+\alpha_{y} e^{-\xi T_{p}}\right)+e^{-2 \xi T_{p}}\right) s+\frac{\gamma \bar{N} e^{-\xi T_{p}}}{\omega_{p}}\left(-e^{-\xi T_{p}}+\alpha_{x}\right)=0 .
$$

In fact, (40) has one solution satisfying $s=1$ for $\bar{N}=\omega_{p} / \gamma=\bar{N}_{\text {cr }}$, while below this threshold we have $|s|<1$.

The whole bifurcation scenario for different values of $\gamma_{2}$ and $\gamma_{3}$ is qualitatively depicted in Figure 3 .

It is worth remarking that, again, the most interesting properties are determined only by the local behavior of $f_{\sigma}\left(A_{0}\right)$.

Up to now we have considered only the case $k=1$, which is the most interesting from a practical point of view because it is the unique case in which the model has a bifurcation point, which describes well, both qualitatively and quantitatively, the real behavior. For the sake of completeness we consider now also the cases $k<1$ and $k>1$. Functions with these characteristics are schematically shown in Figure 2.

By referring to Figure 2 the solution scenarios can be easily understood. For $k<1$, and supposing that $f_{\sigma}\left(A_{0}\right)$ has regular behavior with an always negative curvature (as the function in Figure 2, see point (3)), we see that, in addition to $A_{0}=0$, for every value of $\bar{N}$ there is always one and only one solution $A_{0}$. Furthermore, the function $A_{0}=A_{0}(\bar{N})$ is monotonically increasing and goes to infinity for $\bar{N} \rightarrow \infty$. 


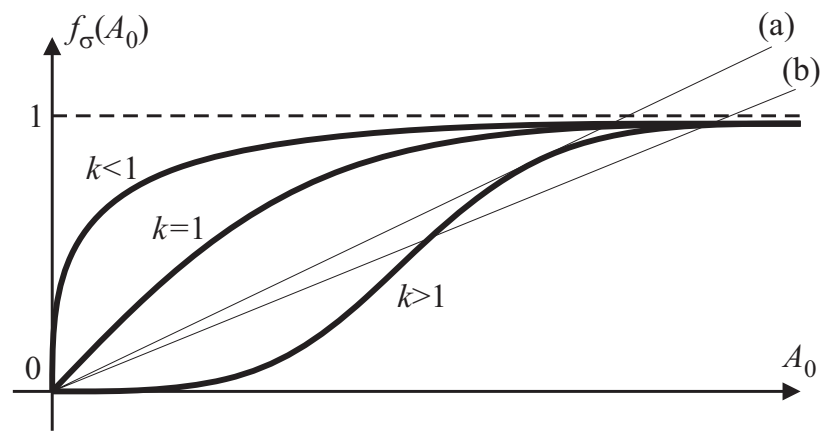

Figure 2. A schematic representation of $f_{\sigma}\left(A_{0}\right)$ for different values of exponent $k$ of (26).

The case $k>1$ is slightly more involved. In fact we have that for small values of $\bar{N}$ (that is, very steep straight lines), there are no solutions. When $\bar{N}$ increases, at a certain threshold the line becomes tangent to the curve $f_{\sigma}\left(A_{0}\right)$ (as line (a) in Figure 2), at a point ahead of the unique inflection point. Above this threshold there are always two solutions (see line (b) in Figure 2), one of which approaches zero and the other infinity as $\bar{N} \rightarrow \infty$. This is a saddle-node bifurcation, where a solution suddenly appears, far from the main path. This does not seem to capture the behavior observed in real cases, although we cannot exclude in principle that it could happen in different (unobserved up to now) situations. We only note that to detect the saddle-node threshold a local analysis around $A_{0}=0$ is no longer sufficient.

4.2. Imperfect case. In the imperfect case $f_{\sigma}(0)=\varepsilon>0$, so that $A_{0}=0$ is no longer a trivial solution. Considering the most interesting case $k=1$ we have that

$$
\frac{\omega_{p}}{\bar{N}} A_{0}=\varepsilon+\gamma A_{0}+\gamma_{2}\left(A_{0}\right)^{2}+\gamma_{3}\left(A_{0}\right)^{3}+\ldots
$$

so that locally the solution is

$$
\frac{\bar{N}}{\omega_{p}}=\frac{A_{0}}{\varepsilon}-\gamma\left(\frac{A_{0}}{\varepsilon}\right)^{2}+\left(-\gamma_{2} \varepsilon+\gamma^{2}\right)\left(\frac{A_{0}}{\varepsilon}\right)^{3}-\left(\gamma_{3}^{2}-2 \gamma \gamma_{2} \varepsilon+\gamma^{3}\right)\left(\frac{A_{0}}{\varepsilon}\right)^{4}+\ldots
$$

The main branch emanating from $\left(A_{0}, \bar{N}\right)=(0,0)$ is no longer at rest, although not so far from it, since $\varepsilon$ is small - otherwise it cannot be considered as an imperfection and must be carefully considered in an appropriate way. There are no longer branching points and branching paths ensuing from the main one, a fact that constitutes the main distinction with respect to the perfect case (Section 4.1).

All possible situations are qualitatively depicted in Figure 3, together with the corresponding perfect scenario. Each case of Figure 3 is clearly an unfolding of a local branching bifurcation, according to the fact that transcritical and pitchfork bifurcations are not structurally stable [Wiggins 1997].

Comparing the pictures of Figure 3 with the numerical simulations of the SAMEO model [Marcheggiani and Lenci 2010] and with the experimental outcomes (the results of the Arup tests can be looked up, for example, in [Newland 2001; Abrams 2006]) we see that the situation actually occurring is that of Figure 3c; in fact, for low values of $N$ there are small (but not null) oscillations, which suddenly but not instantaneously (as it would be in the perfect case of a pitchfork bifurcation) increase around a critical threshold. 

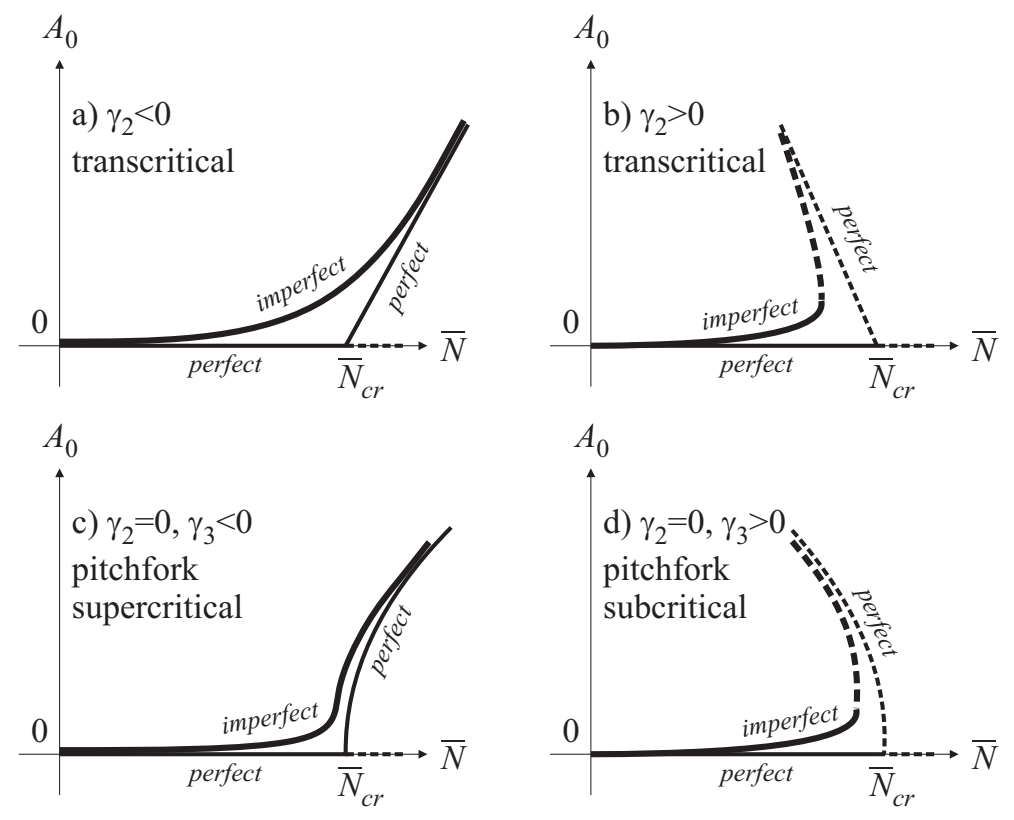

Figure 3. Qualitative bifurcation paths for perfect and imperfect cases; stable (solid lines) and unstable (dashed lines).

From the previous considerations we can draw the following conclusions:

- The theoretical critical value $N_{\mathrm{cr}}$ computed in the previous section is a reference value, of course of great engineering interest, and not the mathematically exact value of the critical threshold, which actually does not exist.

- The dynamical phenomenon underlying the problem of pedestrian-induced lateral vibrations of footbridges is a perturbation of a pitchfork bifurcation. This result was also obtained in [Lenci and Marcheggiani 2008] and is herein confirmed with a different model.

\section{The resonant case}

We have seen in the previous sections that the resonant case is the worst situation, and thus in this section it is studied in detail.

We start by noticing that the minimum of $N_{\mathrm{cr}}$ for varying $\omega_{p}$ is obtained for $\omega_{p}=\sqrt{1-2 \xi^{2}}$ (see (34)). This is the mathematical resonance, corresponding to the maximum of the amplification factor [Clough and Penzien 1975]. The engineering resonance is given by $\omega_{p}=\sqrt{1-\xi^{2}}$ and corresponds to the coincidence between the external (excitation) and internal (natural) frequencies. When $\xi$ is small, as occurs in practical cases, the difference is negligible.

In the engineering resonance case we have $\alpha_{x}=\alpha_{y}=1, \alpha_{x y}=0$, and the map given by (22) and (25) becomes $\left(T_{p}=2 \pi / \sqrt{1-\xi^{2}}\right)$ :

$$
x_{n+1}=e^{-\xi T_{p}} x_{n}, \quad y_{n+1}=e^{-\xi T_{p}} y_{n}+\sigma_{n} \bar{N}\left(1-e^{-\xi T_{p}}\right), \quad \sigma_{n+1}=f_{\sigma}\left(A_{n}\right) .
$$


From (43) $)_{1}$ we conclude that, for any trajectory, $x_{n} \rightarrow 0$ for $n \rightarrow \infty$. This means that the (planar) invariant manifold $x=0$ is globally attractive, so that the most interesting dynamics live on it. Note that due to $(28)_{1}$ the fixed points obtained in the previous section belong to the manifold.

On the invariant manifold system (43) reduces to the 2D map

$$
y_{n+1}=(1-a) y_{n}+a \bar{N} \sigma_{n}, \quad \sigma_{n+1}=f_{\sigma}\left(\frac{\left|y_{n}\right|}{\omega_{p}}\right)=f_{\sigma}\left(\frac{\left|y_{n}\right|}{\sqrt{1-\xi^{2}}}\right)=\bar{f}_{\sigma}\left(\left|y_{n}\right|\right),
$$

where $a=1-e^{-\xi T_{p}}=1-e^{-2 \pi \xi / \sqrt{1-\xi^{2}}}$ is a positive number less than 1 . In real structures it is possibly small, $a \cong 2 \pi \xi$ (and in this case $f_{\sigma}$ coincides with $\bar{f}_{\sigma}$ ), but this hypothesis is not required here.

Given an initial point $P_{n}=\left(y_{n}, \sigma_{n}\right)$ in the phase space $(\mathbb{R},[0,1])$ of $(44)$, its image $P_{n+1}=\left(y_{n+1}, \sigma_{n+1}\right)$ can be obtained by the following graphical procedure, which is a noticeable property of map (44) and which is illustrated in Figure 4:

(1) From $P_{n}$ draw a vertical line and individuate points $A$ and $B$ where it intersects line $r$ of equation $\sigma_{n}=y_{n} / \bar{N}$ and the function $\bar{f}_{\sigma}\left(\left|y_{n}\right|\right)$, respectively.

(2) From $A$ draw the line $s$ of slope $1 /(a \bar{N})$ (which is more steep than line $r$ since $a<1$ ).

(3) From $P_{n}$ draw a horizontal line and individuate the point $C$ of intersection with $s$.

(4) From $C$ draw a vertical line and from $B$ a horizontal line. The intersection point is $P_{n+1}$.

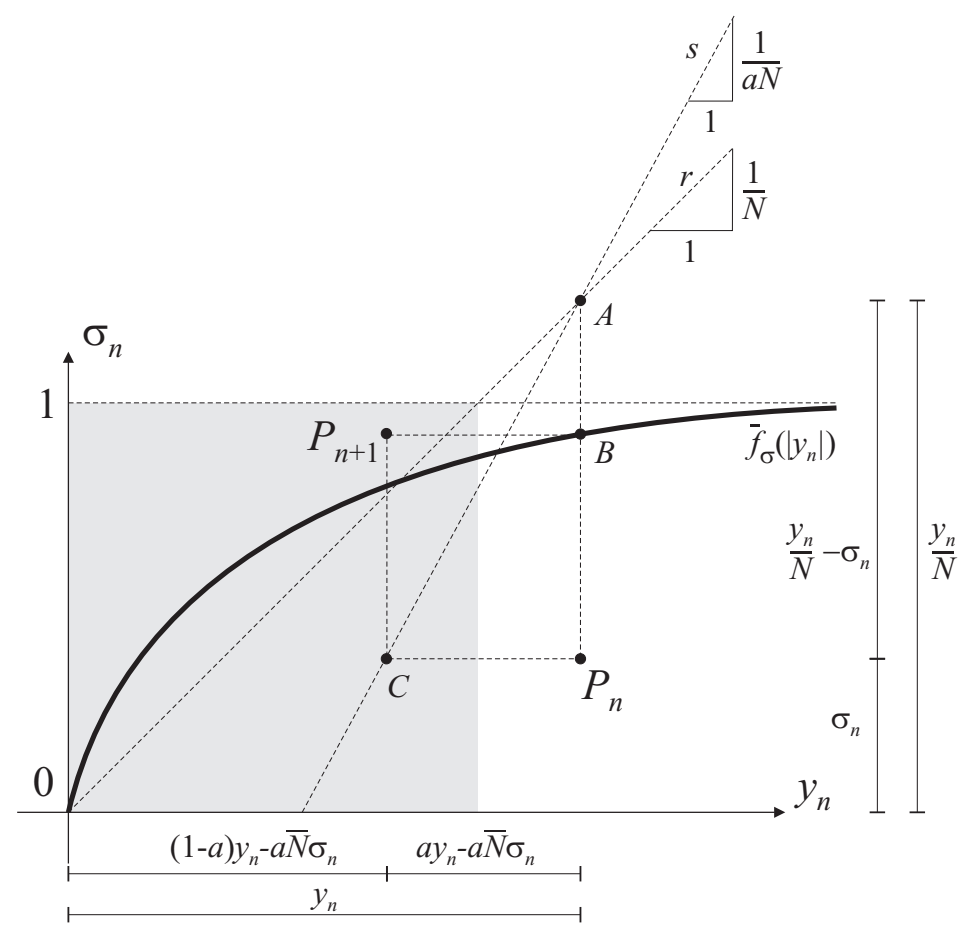

Figure 4. Sketch of the graphical construction of the 2D map (44). In gray is attracting region $R=\left(y_{n}, \sigma_{n}\right) \in([0, \bar{N}],[0,1])$. 


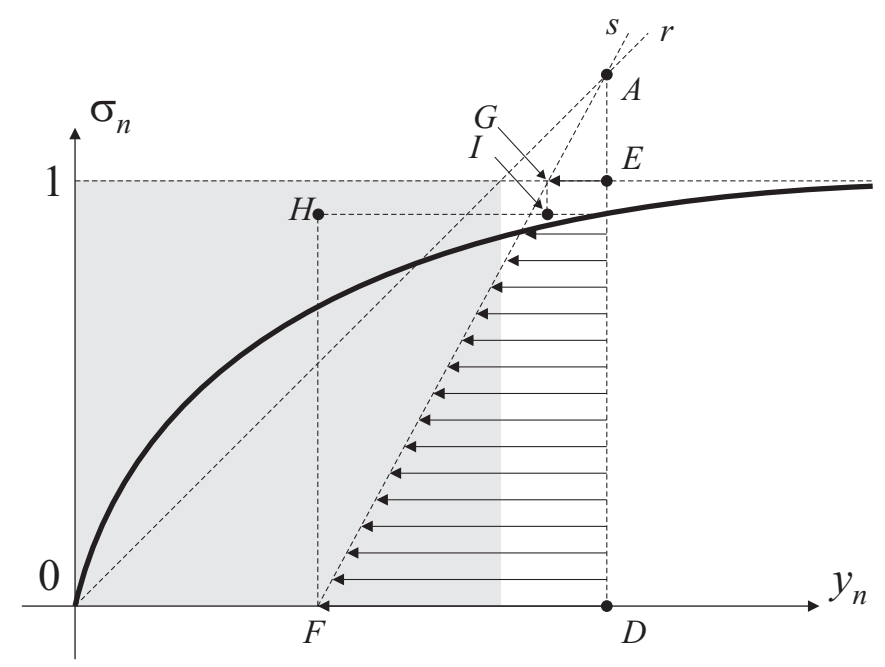

Figure 5. Some properties of the map (44).

From the previous graphical construction it is immediately seen that the unique nontrivial fixed point (when it exists, that is, above $\bar{N}_{\mathrm{cr}}$ ) corresponds to $y_{0}$ such that points $A$ and $B$ coincide, that is, when line $r$ intersects $\bar{f}_{\sigma}\left(\left|y_{n}\right|\right)$. This corresponds exactly to the point found by the graphical construction of Figure 1.

By using the graphical construction of Figure 4 it is possible to note the following properties, which are illustrated in Figure 5 and which further help in understanding the behavior of the 2D map:

- segment $F-G$ is the diagram of the horizontal displacements of the points belonging to segment $D-E$, that is, the points having a fixed $y_{n}$ and varying $\sigma_{n}$ and

- the image of segment $D-E$ is segment $H-I$.

It is useful to rewrite $(44)_{1}$ in the alternative form

$$
\Delta y_{n}=y_{n+1}-y_{n}=a\left(\bar{N} \sigma_{n}-y_{n}\right) .
$$

Since $0 \leq \sigma_{n} \leq 1$, we have that

$$
-a y_{n} \leq \Delta y_{n} \leq a\left(\bar{N}-y_{n}\right)
$$

From the left-hand side inequality we see that for all negative $y_{n}$ the difference $\Delta y_{n}$ is positive, so that every point in the region $y_{n}<0$ tends to move toward $y_{n}=0$. From the right-hand side inequality, on the other hand, we see that for all $y_{n}>\bar{N}$ the difference $\Delta y_{n}$ is negative, so that every point in the region $y_{n}>\bar{N}$ tends to move toward $y_{n}=\bar{N}$ (see an example in Figure 5). The conclusion is that the region $R=\left(y_{n}, \sigma_{n}\right) \in([0, \bar{N}],[0,1])$, which is shown in gray in Figures 4 and 5 , is globally attracting, and the steady-state behavior lies therein. In fact, points belonging to $R$ do not escape from it, since from $0 \leq y_{n} \leq \bar{N}$ and $0 \leq \sigma_{n} \leq 1$ it follows (see (44) 1 ) that $0 \leq y_{n+1} \leq \bar{N}$.

On the attracting region $R$ the map (44) is invertible, because $\bar{f}_{\sigma}\left(y_{n}\right)$ is invertible on $\mathbb{R}^{+}$by the assumptions made in Section 3. 


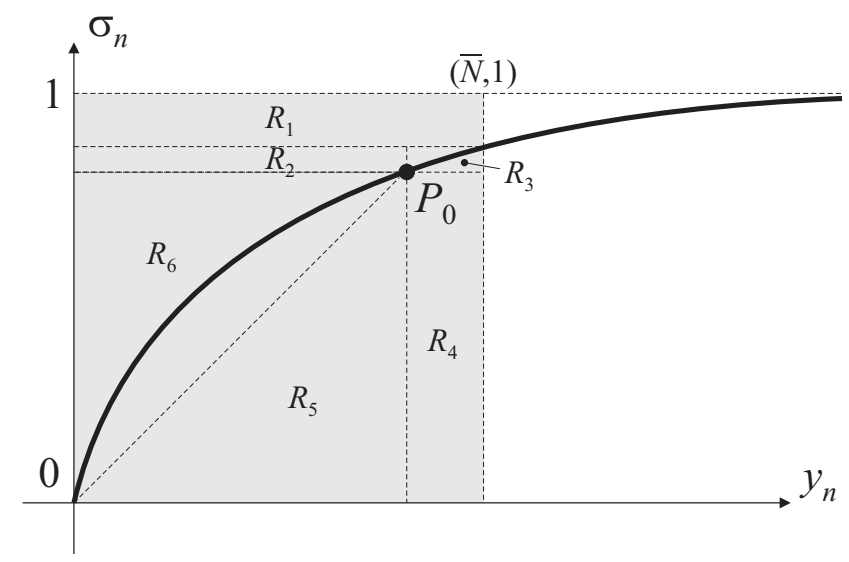

Figure 6. The six subregions of the attracting region of the map (44).

In the case $\bar{N}>\bar{N}_{\text {cr }}$ the region $R$ can be divided into six subregions, as shown in Figure 6. From the graphical constructions introduced above we see that:

- $R_{1} \rightarrow R_{3} \cup R_{4} \cup R_{5} \cup R_{6}$;

- $R_{2} \rightarrow R_{4} \cup R_{5} \cup R_{6}$;

- $R_{3} \rightarrow R_{3}$;

- $R_{4} \rightarrow R_{2} \cup R_{3}$;

- $R_{5} \rightarrow R_{6}$

- $R_{6} \rightarrow R_{5} \cup R_{6}$.

An example is reported in Figure 7 where $\bar{f}_{\sigma}(y)=\tanh (y), \bar{N}=1.5\left(>\bar{N}_{\text {cr }}=1\right)$, and $a=0.3$.

From the previous scheme we conclude that periodic or chaotic solutions (not necessarily stable) are possible only in $R_{3}$, alternating in $R_{2}$ and $R_{4}$, and alternating in $R_{5}$ and $R_{6}$. These considerations are the starting point for the detailed study of the dynamical behavior of the map (44), which is out of the
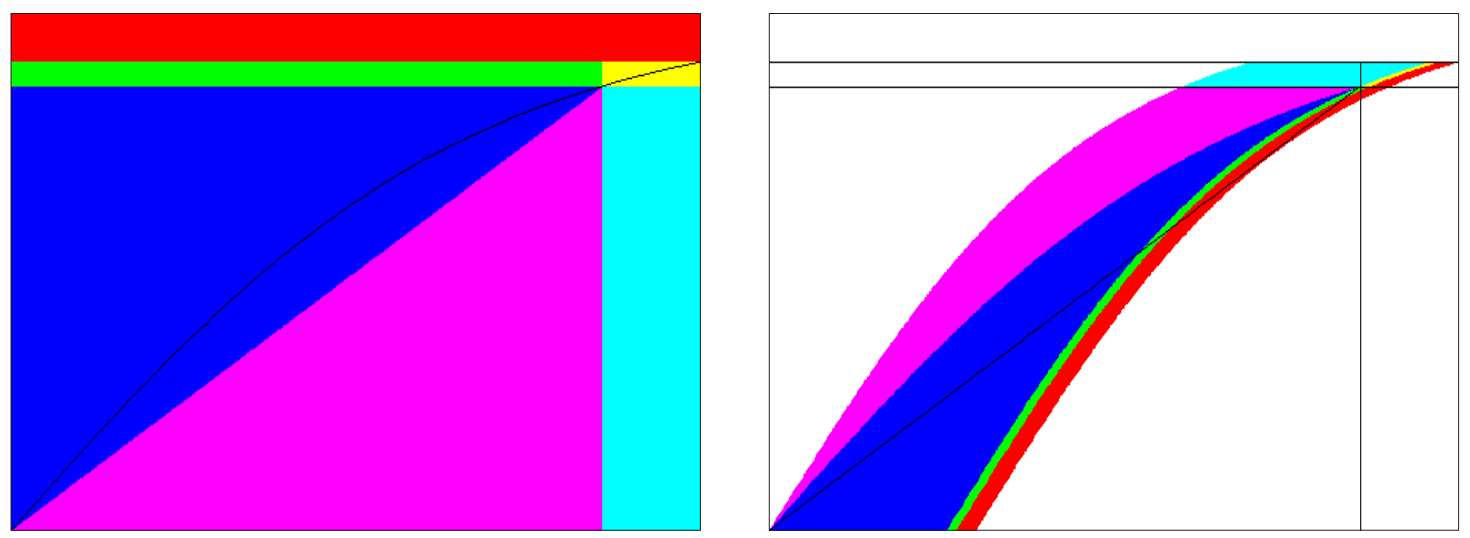

Figure 7. The six subregions of the attracting region and their images after one iteration of the map, where $\bar{f}_{\sigma}(y)=\tanh (y), \bar{N}=1.5\left(>\bar{N}_{\text {cr }}=1\right)$, and $a=0.3$. 
scope of the present paper and is left for future work. We only note that in some isolated numerical simulations based on the example of Figure 7 we have seen that the bifurcated equilibrium point $P_{0}$ is globally attractive for the whole phase space.

\section{Conclusions}

A 3D discrete-time dynamical system has been proposed for studying the pedestrian-induced lateral vibrations of footbridges. For the mechanical part, the model is based on the stroboscopic Poincaré map of the flow of the associated continuous time, one mode system, while the coupling between the pedestrians and the bridge motion has been modeled by assuming that the degree of synchronization of the pedestrians is a function $f_{\sigma}$ of the amplitude of the bridge oscillations.

The fixed points of the map have been studied in detail, without exact knowledge of $f_{\sigma}$. Only the local behavior of $f_{\sigma}$ around the rest position and some qualitative properties have been used. Both the perfect and the imperfect cases have been considered.

In the perfect case it has been shown that in the unique case of interest in practice (corresponding to $\left.f_{\sigma}^{\prime}(0)=\gamma \in\right] 0, \infty[)$ there is a main path of rest solutions. When the number of pedestrians $N$ increases, at a certain threshold $N_{\mathrm{cr}}$ a secondary path bifurcates from the previous one, thus allowing for "large" oscillations of the bridge. This is the threshold of activation of the unwanted lateral oscillations, and it is of primary importance in practice. With the proposed model a very simple, predictive, and general formula is obtained for $N_{\mathrm{cr}}$, a fact that constitutes the main result of this paper.

The imperfect case has been considered, by including the effect of small imperfections. It has been shown how the four possible fixed-points scenarios are modified by the imperfections. The one corresponding to a perturbation of a pitchfork bifurcation is noted to agree with experimental observations on real cases (the Arup experiments on the Millennium Bridge) and with numerical simulations of a more sophisticated model. Thus, it is concluded that the dynamical phenomenon underlying the synchronization problem is a perturbation of the pitchfork bifurcation.

The present paper is devoted to the construction of the model and to the study of the fixed points, which is sufficient to obtain the desired formula for the critical number of pedestrians and to understand the main dynamical aspects. The detailed study of the whole dynamics of the system, including more complex phenomena such as chaos, is worthwhile but out of the scope of this work, and is left for future work.

\section{Acknowledgements}

Lenci thanks the Organizing Committee of PACAM XI, and its President Adair Aguiar, for supporting his participation in the Congress. Thanks are also gratefully due to Carlos Mazzilli and Paulo Gonçalves for supporting a post-Congress visit at USP and PUC, during which part of this work has been developed.

\section{References}

[Abrams 2006] D. M. Abrams, Two coupled oscillator models: the Millennium Bridge and the Chimera state, Ph.D. thesis, Theoretical and Applied Mechanics, Cornell University, Ithaca, NY, 2006, available at http://hdl.handle.net/1813/3271.

[Arup 2000] Arup Group, “The Millennium Bridge”, 2000. http://www.arup.com/MillenniumBridge/indepth/video.html. 
[Belli et al. 2001] A. Belli, P. Bui, A. Berger, A. Geyssant, and J.-R. Lacour, "A treadmill ergometer for three-dimensional ground reaction forces measurement during walking", J. Biomech. 34:1 (2001), 105-112.

[Blanchard et al. 1977] J. Blanchard, B. L. Davies, and J. W. Smith, "Design criteria and analysis for dynamic loading of footbridges", pp. 90-107 in Symposium on Dynamic Behaviour of Bridges (Crowthorne, 1977), TRRL Supplementary Report 275, Transport and Road Research Laboratory, Crowthorne, U.K., 1977.

[Blekherman 2007] A. N. Blekherman, "Autoparametric resonance in a pedestrian steel arch bridge: Solferino bridge, Paris", J. Bridge Eng. 12:6 (2007), 669-676.

[Bodgi et al. 2007] J. Bodgi, S. Erlicher, and P. Argoul, "Lateral vibration of footbridges under crowd-loading: continuous crowd modeling approach", Key Eng. Mater. 347 (2007), 685-690.

[Candaten and Rinaldi 2000] M. Candaten and S. Rinaldi, "Peak-to-peak dynamics: a critical survey", Int. J. Bifurc. Chaos 10:8 (2000), 1805-1820.

[Clough and Penzien 1975] R. Clough and J. Penzien, Dynamics of structures, McGraw-Hill, New York, 1975.

[Dallard et al. 2001a] P. Dallard, A. J. Fitzpatrick, A. Flint, S. Le Bourva, A. Low, R. M. R. Smith, and M. Willford, “The London Millennium Footbridge", Struct. Eng. 79:22 (2001), 17-33.

[Dallard et al. 2001b] P. Dallard, A. J. Fitzpatrick, A. Flint, A. Low, R. M. R. Smith, M. Willford, and M. Roche, "London Millennium Bridge: pedestrian-induced lateral vibration”, J. Bridge Eng. 6:6 (2001), 412-417.

[Danbon and Grillaud 2005] F. Danbon and G. Grillaud, "Dynamic behaviour of a steel footbridge: characterization and modelling of the dynamic loading induced by a moving crowd on the Solferino Footbridge in Paris", in Proceedings of Footbridge 2005: 2nd International Conference (Venice, 2005), Office Technique pour l'Utilisation de l'Acier, Puteaux, 2005.

[Eckhardt et al. 2007] B. Eckhardt, E. Ott, S. H. Strogatz, D. M. Abrams, and A. McRobie, "Modeling walker synchronization on the Millennium Bridge", Phys. Rev. E 75:2 (2007), 021110.

[Fujino et al. 1993] Y. Fujino, B. M. Pacheco, S.-I. Nakamura, and P. Warnitchai, "Synchronization of human walking observed during lateral vibration of a congested pedestrian bridge”, Earthq. Eng. Struct. Dyn. 22:9 (1993), 741-758.

[Johansson et al. 2008] A. Johansson, D. Helbing, H. Z. Al-Abideen, and S. Al-Bosta, "From crowd dynamics to crowd safety: a video-based analysis", Adv. Complex Syst. 11:4 (2008), 497-527.

[Lenci and Marcheggiani 2008] S. Lenci and L. Marcheggiani, "A discrete-time model for the phenomenon of synchronous lateral excitation due to pedestrians motion on footbridges", in Proceedings of Footbridge 2008: Footbridge for urban renewal: 3rd International Conference (Porto, 2008), edited by E. de Sá Caetano and Á. Cunha, University of Porto, College of Engineering (FEUP), Porto, 2008.

[Marcheggiani and Lenci 2010] L. Marcheggiani and S. Lenci, "On a model for the pedestrians-induced lateral vibrations of footbridges", Meccanica (Milano) 45:4 (2010), 531-551.

[Matsumoto et al. 1978] Y. Matsumoto, T. Nishioka, H. Shiojiri, and K. Matsuzaki, "Dynamic design of footbridges", IABSE Proc. 2 (1978), 1-15. Paper P-17/78.

[Nakamura 2004] S.-I. Nakamura, "Model for lateral excitation of footbridges by synchronous walking", J. Struct. Eng. (ASCE) 130:1 (2004), 32-37.

[Nakamura and Kawasaki 2006] S.-I. Nakamura and T. Kawasaki, "Lateral vibration of footbridges by synchronous walking", J. Constr. Steel Res. 62:11 (2006), 1148-1160.

[Nakamura and Kawasaki 2009] S.-I. Nakamura and T. Kawasaki, "A method for predicting the lateral girder response of footbridges induced by pedestrians", J. Constr. Steel Res. 65:8-9 (2009), 1705-1711.

[Newland 2001] D. E. Newland, "Vibration: problem and solution", pp. 88-93 in Blade of light: the story of London's Millennium Bridge, edited by D. Sudjic, Penguin/Millennium Bridge Trust, London, 2001.

[Newland 2003] D. E. Newland, "Pedestrian excitation of bridges: recent results", pp. 1-15 in Proceedings of the Tenth International Congress on Sound and Vibration (ICSV-10) (Stockholm, 2003), edited by A. Nilsson and H. Boden, International Institute of Acoustics and Vibration (IIAV), Stockholm, 2003.

[Piccardo and Tubino 2008] G. Piccardo and F. Tubino, "Parametric resonance of flexible footbridges under crowd-induced lateral excitation”, J. Sound Vib. 311:1-2 (2008), 353-371. 
[Ricciardelli and Pizzimenti 2007] F. Ricciardelli and A. D. Pizzimenti, "Lateral walking-induced forces on footbridges", $J$. Bridge Eng. 12:6 (2007), 677-688.

[Roberts 2005] T. M. Roberts, "Lateral pedestrian excitation of footbridges", J. Bridge Eng. 10:1 (2005), 107-112.

[Strogatz et al. 2005] S. H. Strogatz, D. M. Abrams, A. McRobie, B. Eckhardt, and E. Ott, "Theoretical mechanics: crowd synchrony on the Millennium Bridge", Nature 438:7064 (2005), 43-44.

[Venuti and Bruno 2009] F. Venuti and L. Bruno, "Crowd-structure interaction in lively footbridges under synchronous lateral excitation: a literature review”, Phys. Life Rev. 6:3 (2009), 176-206.

[Venuti et al. 2007] F. Venuti, L. Bruno, and N. Bellomo, "Crowd dynamics on a moving platform: mathematical modelling and application to lively footbridges", Math. Comput. Model. 45:3-4 (2007), 252-269.

[Wheeler 1980] J. E. Wheeler, "Pedestrian induced vibration in footbridges", Technical report 15, Main Roads Department, Perth, 1980.

[Wiggins 1997] S. Wiggins, Introduction to applied nonlinear dynamical systems and chaos, Texts in Applied Mathematics 2 , Springer, New York, 1997.

[Živanović et al. 2005] S. Živanović, A. Pavic, and P. Reynolds, "Vibration serviceability of footbridges under human-induced excitation: a literature review", J. Sound Vib. 279:1-2 (2005), 1-74.

Received 23 Jun 2010. Revised 26 Nov 2010. Accepted 30 Nov 2010.

STEFANO LENCI: lenci@univpm.it

Dipartimento di Ingegneria Civile, Edile e Architettura, Università Politecnica delle Marche, via Brecce Bianche, I-60131 Ancona, Italy

LAURA MARCHEGGIANI: 1.marcheggiani@univpm.it

Dipartimento di Ingegneria Civile, Edile e Architettura, Università Politecnica delle Marche, via Brecce Bianche, I-60131 Ancona, Italy 


\title{
JOURNAL OF MECHANICS OF MATERIALS AND STRUCTURES
}

\author{
jomms.org
}

Founded by Charles R. Steele and Marie-Louise Steele

EDITORS

Charles R. SteEle

DAVIDE BIGONI

Stanford University, USA

YASUHIDE SHINDO

University of Illinois at Urbana-Champaign, USA

Tohoku University, Japan

\section{EDITORIAL BOARD}

$\begin{aligned} \text { H. D. BUI } & \text { École Polytechnique, France } \\ \text { J. P. CARTER } & \text { University of Sydney, Australia } \\ \text { R. M. CHRISTENSEN } & \text { Stanford University, USA } \\ \text { G. M. L. GLADWELL } & \text { University of Waterloo, Canada } \\ \text { D. H. HODGES } & \text { Georgia Institute of Technology, USA } \\ \text { J. HUTCHINSON } & \text { Harvard University, USA } \\ \text { C. HWU } & \text { National Cheng Kung University, Taiwan } \\ \text { B. L. KARIHALOO } & \text { University of Wales, UK } \\ \text { Y. Y. KIM } & \text { Seoul National University, Republic of Korea } \\ \text { Z. MROZ } & \text { Academy of Science, Poland } \\ \text { D. PAMPLONA } & \text { Universidade Católica do Rio de Janeiro, Brazil } \\ \text { M. B. RUBIN } & \text { Technion, Haifa, Israel } \\ \text { A. N. SHUPIKOV } & \text { Ukrainian Academy of Sciences, Ukraine } \\ \text { T. TARNAI } & \text { University Budapest, Hungary } \\ \text { F. Y. M. WAN } & \text { University of California, Irvine, USA } \\ \text { P. WRIGGERS } & \text { Universität Hannover, Germany } \\ \text { W. YANG } & \text { Tsinghua University, China } \\ \text { F. ZIEGLER } & \text { Technische Universität Wien, Austria } \\ & \\ \text { PRODUCTION } & \text { contact@ msp.org } \\ \text { SILVIO LEVY } & \text { Scientific Editor }\end{aligned}$

Cover design: Alex Scorpan

Cover photo: Mando Gomez, www.mandolux.com

See http://jomms.org for submission guidelines.

JoMMS (ISSN 1559-3959) is published in 10 issues a year. The subscription price for 2011 is US \$520/year for the electronic version, and \$690/year (+\$60 shipping outside the US) for print and electronic. Subscriptions, requests for back issues, and changes of address should be sent to Mathematical Sciences Publishers, Department of Mathematics, University of California, Berkeley, CA 94720-3840.

JoMMS peer-review and production is managed by EditFLow ${ }^{\circledR}$ from Mathematical Sciences Publishers.

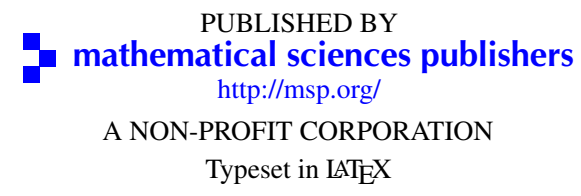

Copyright (C2011 by Mathematical Sciences Publishers 


\section{Journal of Mechanics of Materials and Structures}

\section{Volume 6, No. 7-8}

September-October 2011

\section{Special issue \\ Eleventh Pan-American Congress \\ of Applied Mechanics (PACAM XI)}

Preface

Adair R. Aguiar

949

Influence of specimen geometry on the Portevin-Le Châtelier effect due to dynamic strain aging

for the AA5083-H116 aluminum alloy

Rodrigo Nogueira de Codes and Ahmed Benallal

Dispersion relations for SH waves on a magnetoelectroelastic heterostructure with imperfect

interfaces

J. A. Otero, H. Calas, R. Rodríguez, J. Bravo, A. R. Aguiar and G. Monsivais

Numerical linear stability analysis of a thermocapillary-driven liquid bridge with magnetic stabilization

Yue Huang and Brent C. Houchens

Numerical investigation of director orientation and flow of nematic liquid crystals in a planar 1:4 expansion Pedro a. Cruz, Murilo F. Tomé, IAin W. Stewart and Sean McKee

Critical threshold and underlying dynamical phenomena in pedestrian-induced lateral vibrations of footbridges

Stefano LenCI and LAURA MARCHEgGiani

Free vibration of a simulation CANDU nuclear fuel bundle structure inside a tube

XUAN ZHANG and SHUdONG Yu

Nonlinear dynamics and sensitivity to imperfections in Augusti's model

D. Orlando, P. B. Gonçalves, G. Rega and S. LenCi

Active control of vortex-induced vibrations in offshore catenary risers: A nonlinear normal mode approach

CArlos E. N. MAZzilli and César T. SANCheS

Nonlinear electromechanical fields and localized polarization switching of piezoelectric macrofiber composites

Yasuhide Shindo, Fumio Narita, KoJi SATo and Tomo TAKeda

1089

Three-dimensional BEM analysis to assess delamination cracks between two transversely isotropic materials

Nicolás O. Larrosa, Jhonny E. Ortiz and Adrián P. Cisillino

Porcine dermis in uniaxial cyclic loading: Sample preparation, experimental results and modeling

A. E. Ehret, M. Hollenstein, E. MAzzA and M. Itskov

Analysis of nonstationary random processes using smooth decomposition

Rubens SAMpaio and Sergio Bellizzi

Perturbation stochastic finite element-based homogenization of polycrystalline materials

S. LePage, F. V. Stump, I. H. Kim and P. H. Geubelle

A collocation approach for spatial discretization of stochastic peridynamic modeling of fracture

Georgios I. Evangelatos and POL D. SPANOS 